\title{
ON THE METHOD OF STRAINED PARAMETERS AND THE METHOD OF AVERAGING*
}

\author{
BY \\ D. P. MASON \\ University of the Witwatersrand, Johannesburg
}

\begin{abstract}
The method of multiple scales can be modified to remove remaining undesirable features in the perturbation solution by expanding available parameters in the equation(s) (Veronis [1]). Corresponding modifications to the Lindstedt-Poincare method of strained parameters and the method of averaging are investigated and illustrated using the Duffing equation. It is found that a solution to the Duffing equation with no secular terms in either the amplitude or the frequency can be obtained simply by expanding the parameter in the equation without making the near-identity transformation of the independent variable associated with the Lindstedt-Poincare technique.
\end{abstract}

1. Introduction. Veronis [1] has shown that by expanding available parameters in the equation(s) in powers of the perturbation parameter $\varepsilon$, the method of multiple scales can be modified to remove remaining undesirable features of the perturbation solution, such as secular behaviour in the frequency. With the derivative expansion variant of the method of multiple scales, when applied to the Duffing equation for instance, two expansions besides that of the dependent variable are used in this modified procedure: the expansion of the derivative to suppress the secular terms in the amplitude and the expansion of the parameter to suppress the secular terms in the frequency.

In this paper we investigate how the Lindstedt-Poincare method of strained parameters and the method of averaging can similarly be modified to yield more accurate perturbation solutions. Both the standard Krylov-Bogoliubov procedure $[2,3]$ and the generalized method of averaging as developed by Bogolibuov and Mitropolski [2, 4] which allows higher order terms to be calculated, are considered. The techniques are illustrated by the Duffing equation

$$
\dot{u}+\omega^{2} u=-\varepsilon u^{3}
$$

where an overhead dot denotes differentiation with respect to time $t$, and $\omega$ is a parameter. All three techniques (the method of multiple scales, the Lindstedt-Poincare method of strained parameters and the method of averaging) can be modified to yield uniformly valid solutions to Eq. (1.1), and hence the procedures and results can be compared.

* Received by the editors February 8, 1983. 
2. Lindstedt-Poincaré method of strained parameters. In the Lindstedt-Poincaré method the new independent variable $s$ is introduced through the near-identity transformation $s=\Omega t$ and $\Omega$ and $u$ are expanded according to

$$
\begin{gathered}
\Omega=\omega+\varepsilon \omega_{1}+\varepsilon^{2} \omega_{2}+\cdots, \\
u(t ; \varepsilon)=u_{0}(s)+\varepsilon u_{1}(s)+\varepsilon^{2} u_{2}(s)+\cdots,
\end{gathered}
$$

where the constants $\omega_{n}$ for $n \geqslant 1$ are chosen, as the solution develops, to remove the secular behaviour in the amplitude. It is easily verified that for the Duffing equation this method gives

$$
\begin{aligned}
u(t ; \varepsilon)= & a_{0} \cos \left(\Omega t+\alpha_{0}\right)+\frac{\varepsilon a_{0}^{3}}{32 \omega^{2}} \cos \left(3 \Omega t+3 \alpha_{0}\right) \\
& +\frac{\varepsilon^{2} a_{0}^{5}}{1024 \omega^{4}}\left[\cos \left(5 \Omega t+5 \alpha_{0}\right)-21 \cos \left(3 \Omega t+3 \alpha_{0}\right)\right]+O\left(\varepsilon^{3}\right),
\end{aligned}
$$

where

$$
\Omega=\omega+\frac{3 a_{0}^{2}}{8 \omega} \varepsilon-\frac{15 a_{0}^{4}}{256 \omega^{3}} \varepsilon^{2}+O\left(\varepsilon^{3}\right),
$$

and $a_{0}$ and $\alpha_{0}$ are constants. In deriving (2.3), no initial conditions were imposed and the homogeneous solution in $u_{n}$ for $n \geqslant 1$ was disregarded. Initial conditions can be imposed on (2.3) by treating the constants $a_{0}$ and $\alpha_{0}$ as functions of $\varepsilon$ and by expanding them in powers of $\varepsilon$. We will follow this procedure throughout the paper since it involves less algebra. It is equivalent to the alternative procedure whereby $u_{n}$ for $n \geqslant 1$ contain the homogeneous solution with two arbitrary constants, $a_{n}$ and $\alpha_{n}$, and the constants are taken to be independent of $\varepsilon[5,6]$.

As pointed out by Veronis [1], (2.3) still contains a secular type behaviour since the frequency term is not uniformly valid, being a power series of terms multiplying $t$. Since the source of the nonuniformity is associated with the frequency, Veronis [1] modified the method of multiple scales by making the additional expansion

$$
\omega^{2}=\omega_{0}^{2}+\varepsilon \omega_{2}+\varepsilon^{2} \omega_{1}+\cdots
$$

where the constants $\omega_{n}$ for $n \geqslant 1$ are chosen to remove secular terms in the frequency; $\omega_{0}$ is then obtained by solving Eq. (2.5) to the appropriate order in $\varepsilon$. We will now show that expansion (2.5) itself is sufficient to remove the secular terms in both the amplitude and the frequency; with the Duffing equation it is not necessary to make the transformation $s=\Omega t$ as in the Lindstedt-Poincare method (or to expand the derivative as in the method of multiple scales).

Substitute (2.5) into Eq. (1.1) and expand

$$
u(t ; \varepsilon)=u_{0}(t)+\varepsilon u_{1}(t)+\varepsilon^{2} u_{2}(t)+\cdots .
$$

Eq. (1.1) becomes, correct to $O\left(\varepsilon^{2}\right)$,

$$
\ddot{u}_{0}+\varepsilon \ddot{u}_{1}+\varepsilon^{2} \ddot{u}_{2}+\left(\omega_{0}^{2}+\varepsilon \omega_{1}+\varepsilon^{2} \omega_{2}\right)\left(u_{0}+\varepsilon u_{1}+\varepsilon^{2} u_{2}\right)=-\varepsilon\left(u_{0}+\varepsilon u_{1}\right)^{3} \text {. }
$$

(i) Zero order in $\varepsilon$. 
The terms independent of $\varepsilon$ in Eq. (2.7) give

$$
\ddot{u}_{0}+\omega_{0}^{2} u_{0}=0 \text {. }
$$

The general solution of Eq. (2.8) is

$$
u_{0}(t)=a_{0} \cos \left(\omega_{0} t+\alpha_{0}\right)
$$

where $a_{0}$ and $\alpha_{0}$ are constants.

(ii) First order in $\varepsilon$.

On equating the coefficients of $\varepsilon$ in Eq. (2.7) we obtain

$$
\ddot{u}_{1}+\omega_{0}^{2} u_{1}=-u_{0}^{3}-\omega_{1} u_{0} .
$$

Using (2.9), Eq. (2.10) can be written as

$$
\ddot{u}_{1}+\omega_{0}^{2} u_{1}=-a_{0}\left(\omega_{1}+\frac{3 a_{0}^{2}}{4}\right) \cos \left(\omega_{0} t+\alpha_{0}\right)-\frac{a_{0}^{3}}{4} \cos \left(3 \omega_{0} t+3 \alpha_{0}\right) .
$$

In order to suppress secular behaviour in the amplitude we insist that the coefficient of $\cos \left(\omega_{0} t+\alpha_{0}\right)$ on the right-hand side of Eq. (2.11) be zero; thus $\omega_{1}=-3 a_{0}^{2} / 4$. With the remaining terms in (2.11) we obtain the particular solution

$$
u_{1}(t)=\frac{a_{0}^{3}}{32 \omega_{0}^{2}} \cos \left(3 \omega_{0} t+3 \alpha_{0}\right) .
$$

(iii) Second order in $\varepsilon$.

Equating the coefficients of $\varepsilon^{2}$ in Eq. (2.7), we obtain

$$
\ddot{u}_{2}+\omega_{0}^{2} u_{2}=-3 u_{0}^{2} u_{1}-\omega_{1} u_{1}-\omega_{2} u_{0},
$$

which, with the aid of (2.9) and (2.12), can be rewritten as

$$
\begin{aligned}
\ddot{u}_{2}+\omega_{0}^{2} u_{2}= & -a_{0}\left(\omega_{2}+\frac{3 a_{0}^{4}}{128 \omega_{0}^{2}}\right) \cos \left(\omega_{0} t+\alpha_{0}\right) \\
& -\frac{3 a_{0}^{5}}{128 \omega_{0}^{2}}\left[\cos \left(3 \omega_{0} t+3 \alpha_{0}\right)+\cos \left(5 \omega_{0} t+5 \alpha_{0}\right)\right] .
\end{aligned}
$$

To suppress the secular terms in the amplitude we choose $\omega_{2}=-3 a_{0}^{4} / 128 \omega_{0}^{2}$. With the remaining terms in Eq. (2.14), we obtain the particular solution

$$
u_{2}(t)=\frac{a_{0}^{5}}{1024 \omega_{0}^{4}}\left[3 \cos \left(3 \omega_{0} t+3 \alpha_{0}\right)+\cos \left(5 \omega_{0} t+5 \alpha_{0}\right)\right] .
$$

Hence the solution, correct to $O\left(\varepsilon^{2}\right)$, is

$$
\begin{aligned}
u(t ; \varepsilon)= & a_{0} \cos \left(\omega_{0} t+\alpha_{0}\right)+\frac{\varepsilon a_{0}^{3}}{32 \omega_{0}^{2}} \cos \left(3 \omega_{0} t+3 \alpha_{0}\right) \\
& +\frac{\varepsilon^{2} a_{0}^{5}}{1024 \omega_{0}^{4}}\left[3 \cos \left(3 \omega_{0} t+3 \alpha_{0}\right)+\cos \left(5 \omega_{0} t+5 \alpha_{0}\right)\right]
\end{aligned}
$$


where

$$
\omega^{2}=\omega_{0}^{2}-\frac{3 a_{0}^{2}}{4} \varepsilon-\frac{3 a_{0}^{4}}{128 \omega_{0}^{2}} \varepsilon^{2} .
$$

On solving (2.17) for $\omega_{0}$ we obtain correct to $O\left(\varepsilon^{2}\right)$,

$$
\omega_{0}=\left(\omega^{2}+\frac{3 a_{0}^{2}}{4} \varepsilon+\frac{3 a_{0}^{4}}{128 \omega^{2}} \varepsilon^{2}\right)^{1 / 2} .
$$

The solution (2.16) with (2.18) agrees with that obtained by Veronis [1] using a modified derivative expansion method of multiple scales. In particular there are no scalar terms in the frequency. It is easily verified that if $\omega_{0}$ given by Eq. (2.18) is expanded in powers of $\varepsilon$ and the expansion is substituted into (2.16), then (2.3) and (2.4) obtained by the Lindstedt-Poincare technique are rederived. The method outlined here is more direct than Veronis's modification of the method of multiple scales and gives the solution with less algebra. However it is applicable to a smaller class of problems; for example, like the Lindstedt-Poincare technique, it cannot treat successfully the problem of the linear oscillator with viscous damping [6].

3. Krylov-Bogoliubov method of averaging. For the Duffing equation, the standard Krylov-Bogoliubov procedure gives the Lindstedt-Poincaré solution (2.3) and (2.4) to $O(1)$ in the amplitude and $O(\varepsilon)$ in the frequency. We first consider here how the procedure can be modified to remove the secular terms in the frequency, before considering the generalized method of averaging in the next section.

We again expand $\omega^{2}$ as in (2.5) and substitute this expansion to $O(\varepsilon)$ into Eq. (1.1):

$$
\ddot{u}+\left(\omega_{0}^{2}+\varepsilon \omega_{1}\right) u=-\varepsilon u^{3} \text {. }
$$

When $\varepsilon=0$, the general solution of Eq. (3.1) is

$$
u=a \cos \left(\omega_{0} t+\alpha\right)
$$

where $a$ and $\alpha$ are constants, and we have

$$
\dot{u}=-\omega_{0} a \sin \left(\omega_{0} t+\alpha\right) \text {. }
$$

When $\varepsilon \neq 0$, it is assumed that the solution of Eq. (3.1) has the same form as (3.2) but with $a$ and $\alpha$ slowly varying functions of $t$. We also assume that (3.3) remains valid. The following two consistency conditions are derived by insisting that the time derivative of (3.2) agrees with (3.3) and that $u(t)$ satisfies Eq. (3.1):

$$
\begin{gathered}
\dot{a} \cos \phi-\dot{\alpha} a \sin \phi=0, \\
\dot{a} \sin \phi+\dot{\alpha} a \cos \phi=\frac{\varepsilon a}{\omega_{0}}\left(a^{2} \cos ^{3} \phi+\omega_{1} \cos \phi\right),
\end{gathered}
$$

where $\phi=\omega_{0} t+\alpha$. The solution of Eqs. (3.4) and (3.5) for $\dot{a}$ and $\dot{\alpha}$ is

$$
\begin{gathered}
\dot{a}=\frac{\varepsilon a}{\omega_{0}}\left(\omega_{1}+a^{2} \cos ^{2} \phi\right) \cos \phi \sin \phi, \\
\dot{\alpha}=\frac{\varepsilon}{\omega_{0}}\left(\omega_{1}+a^{2} \cos ^{2} \phi\right) \cos ^{2} \phi .
\end{gathered}
$$


Eq. (2.6) and (3.7) are averaged in the usual way by operating with

$$
\frac{1}{2 \pi} \int_{0}^{2 \pi} d \phi
$$

keeping $a$ and $\alpha$ constant during the averaging process. We obtain the averaged equations

$$
\begin{gathered}
\dot{a}=0, \\
\dot{\alpha}=\frac{\varepsilon}{2 \omega_{0}}\left(\omega_{1}+\frac{3 a^{2}}{4}\right) .
\end{gathered}
$$

Thus from Eq. (3.8), $a=a_{0}$ where $a_{0}$ is a constant. The right-hand side of Eq. (3.9) is therefore a constant and integration with respect to $t$ gives

$$
\alpha=\frac{\varepsilon}{2 \omega_{0}}\left(\omega_{1}+\frac{3 a_{0}^{2}}{4}\right) t+\alpha_{0}
$$

where $\alpha_{0}$ is a constant. To suppress secular behaviour in the frequency we therefore choose $\omega_{1}=-3 a_{0}^{2} / 4$; thus $\alpha=\alpha_{0}$. We note that $\omega_{1}$ is a constant as required.

The solution (3.2) therefore assumes the form

$$
u(t ; \varepsilon)=a_{0} \cos \left(\omega_{0} t+\alpha_{0}\right)
$$

where

$$
\omega^{2}=\omega_{0}^{2}-\frac{3 a_{0}^{2}}{4} \varepsilon
$$

Solving Eq. (3.12) for $\omega_{0}$ we obtain finally

$$
u(t ; \varepsilon)=a_{0} \cos \left[\left(\omega^{2}+\frac{3 a_{0}^{2} \varepsilon}{4}\right)^{1 / 2} t+\alpha_{0}\right],
$$

which agrees with (2.16) and (2.18). The secular behaviour in the frequency, which occurs in the solution using the standard Krylov-Bogoliubov procedure, has been removed.

In general, the averaged equation for $\dot{\alpha}$ depends on $t$ through $a$ and hence if $a$ is absent from this equation, of if $a$ is a constant, then $\alpha$ will be, except for an additive constant, a constant multiple of $\varepsilon t$ and the solution (3.2) will exhibit secular behaviour in the frequency. One would then choose, if possible, the constant $\omega_{1}$ so that the averaged equation for $\dot{\alpha}$ assumes the form $\dot{\alpha}=0$. If $a$ depends on $t$ and occurs in the averaged equation for $\dot{\alpha}$ then $\alpha$ will no longer be a constant multiple of $\varepsilon t$ and the solution will not necessarily exhibit nonuniformities in the frequency. If such exist however, it would be difficult to remove them using the present procedure because $\omega$ is independent of $t$.

4. Generalized method of averaging. Finally we examine how the generalized method of averaging due to Bogoliubov and Mitropolski $[2,4]$ can be modified to yield a more accurate perturbation solution, again considering the Duffing equation. The standard procedure gives a solution to the Duffing equation which can be expressed in the form (2.3) and (2.4). We will work to terms of $O\left(\varepsilon^{2}\right)$. 
Following the same procedure as in Sec. 3, but including in the coefficient of $u$ in Eq. (3.1) terms to $O\left(\varepsilon^{2}\right)$ in the expansion (2.5), we obtain in place of (3.6) and (3.7) the differential equations

$$
\begin{gathered}
\dot{a}=\frac{\varepsilon a}{\omega_{0}}\left(\omega_{1}+a^{2} \cos ^{2} \phi\right) \cos \phi \sin \phi+\varepsilon^{2} \frac{a \omega_{2}}{\omega_{0}} \cos \phi \sin \phi, \\
\dot{\phi}=\omega_{0}+\frac{\varepsilon}{\omega_{0}}\left(\omega_{1}+a^{2} \cos ^{2} \phi\right) \cos ^{2} \phi+\varepsilon^{2} \frac{\omega_{2}}{\omega_{0}} \cos ^{2} \phi .
\end{gathered}
$$

Instead of averaging Eqs. (4.1) and (4.2) as in Sec. 3, in the generalized method of averaging the near-identity transformation from $(a, \phi)$ to $(\bar{a}, \bar{\phi})$ defined by

$$
\begin{aligned}
& a=\bar{a}+\varepsilon a_{1}(\bar{a}, \bar{\phi})+\varepsilon^{2} a_{2}(\bar{a}, \bar{\phi})+\cdots, \\
& \phi=\bar{\phi}+\varepsilon \phi_{1}(\bar{a}, \bar{\phi})+\varepsilon^{2} \phi_{2}(\bar{a}, \bar{\phi})+\cdots
\end{aligned}
$$

is made, where $a_{n}$ and $\phi_{n}$ for all $n \geqslant 1$ are assumed to be periodic in $\bar{\phi}$ with period $2 \pi$, and it is assumed further that

$$
\begin{gathered}
\frac{d \bar{a}}{d t}=\varepsilon A_{1}(\bar{a})+\varepsilon^{2} A_{2}(\bar{a})+\cdots, \\
\frac{d \bar{\phi}}{d t}=\omega_{0}+\varepsilon B_{1}(\bar{a})+\varepsilon^{2} B_{2}(\bar{a})+\cdots .
\end{gathered}
$$

For the present problem we will see that the $B_{n}$ are constants and that we can modify the procedure by choosing the constants $\omega_{n}$ appropriately as the solution develops so that the $B_{n}$ vanish; this removes the secular terms from the frequency.

With the aid of expansions (4.3) and (4.4) and using also (4.5) and (4.6), the terms of Eqs. (4.1) and (4.2) can be expanded in powers of $\varepsilon$, and hence Eqs. (4.1) and (4.2) provide perturbation equations to each order in $\varepsilon$. These equations are identically satisfied to zero order in $\varepsilon$.

(i) First order in $\varepsilon$.

Equating the coefficients of $\varepsilon$ in the expansion of Eqs. (4.1) and (4.2), we obtain

$$
\begin{gathered}
\omega_{0} \frac{\partial a_{1}}{\partial \bar{\phi}}+A_{1}=\frac{\bar{a}^{3}}{8 \omega_{0}}(2 \sin 2 \bar{\phi}+\sin 4 \bar{\phi})+\frac{\bar{a} \omega_{1}}{2 \omega_{0}} \sin 2 \bar{\phi}, \\
\omega_{0} \frac{\partial \phi_{1}}{\partial \bar{\phi}}+B_{1}=\frac{\bar{a}^{2}}{8 \omega_{0}}(3+4 \cos 2 \bar{\phi}+\cos 4 \bar{\phi})+\frac{\omega_{1}}{2 \omega_{0}}(1+\cos 2 \bar{\phi}) .
\end{gathered}
$$

If we operate on Eqs. (4.7) and (4.8) with

$$
\frac{1}{2 \pi} \int_{0}^{2 \pi} d \bar{\phi}
$$

holding $\bar{a}$ constant when evaluating the integrals, and use the assumption that $a_{1}$ and $\phi_{1}$ are periodic in $\bar{\phi}$ with period $2 \pi$, then a direct calculation gives

$$
A_{1}=0, \quad B_{1}=\frac{1}{2 \omega_{0}}\left(\omega_{1}+\frac{3 \bar{a}^{2}}{4}\right) .
$$

Since $A_{1}=0$, it follows from Eq. (4.5) that correct to $O(\varepsilon), \bar{a}$ is a constant and hence to this order $B_{1}$ is a constant. To suppress secular terms in the frequency we therefore insist 
that $B_{1}=0$ and choose $\omega_{1}=-3 \bar{a}^{2} / 4$; correct to $O(\varepsilon), \omega_{1}$ is a constant as required. Integration with respect to $\bar{\phi}$ of the remaining terms in Eqs. (4.7) and (4.8) gives

$$
\begin{aligned}
& a_{1}(\bar{a}, \bar{\phi})=\frac{\bar{a}^{3}}{32 \omega_{0}^{2}}(2 \cos 2 \bar{\phi}-\cos 4 \bar{\phi}), \\
& \phi_{1}(\bar{a}, \bar{\phi})=\frac{\bar{a}^{2}}{32 \omega_{0}^{2}}(2 \sin 2 \bar{\phi}+\sin 4 \bar{\phi}) .
\end{aligned}
$$

We have disregarded arbitrary additive functions of $\bar{a}$ in (4.10) and (4.11), which essentially corresponds to disregarding the homogeneous solution in $u_{n}$ for $n \geqslant 1$ in Sec. 2 . The solutions of Eqs. (4.5) and (4.6) contain arbitrary constants of integration which can be treated as functions of $\varepsilon$ when imposing the initial conditions. This procedure involves less algebra.

(ii) Second order in $\varepsilon$.

Equating the coefficients of $\varepsilon^{2}$ in the expansion of Eqs. (4.1) and (4.2), we obtain

$$
\begin{aligned}
\omega_{0} \frac{\partial a_{2}}{\partial \bar{\phi}}+A_{2}= & -A_{1} \frac{\partial a_{1}}{\partial \bar{a}}-B_{1} \frac{\partial a_{1}}{\partial \bar{\phi}}+\frac{\bar{a} \phi_{1}}{2 \omega_{0}}\left(\bar{a}^{2}+2 \omega_{1}\right) \cos 2 \bar{\phi}+\frac{\bar{a}^{3} \phi_{1}}{2 \omega_{0}} \cos 4 \bar{\phi} \\
& +\frac{1}{4 \omega_{0}}\left(3 \bar{a}^{2} a_{1}+2 \bar{a} \omega_{2}+2 a_{1} \omega_{1}\right) \sin 2 \bar{\phi}+\frac{3 \bar{a}^{2} a_{1}}{8 \omega_{0}} \sin 4 \bar{\phi}, \\
\omega_{0} \frac{\partial \phi_{2}}{\partial \bar{\phi}}+B_{2}= & -A_{1} \frac{\partial \phi_{1}}{\partial \bar{a}}-B_{1} \frac{\partial \phi_{1}}{\partial \bar{\phi}}+\frac{3 \bar{a} a_{1}}{4 \omega_{0}}+\frac{\omega_{2}}{2 \omega_{0}}+\frac{1}{2 \omega_{0}}\left(\omega_{2}+2 \bar{a} a_{1}\right) \cos 2 \bar{\phi} \\
& +\frac{\bar{a} a_{1}}{4 \omega_{0}} \cos 4 \bar{\phi}-\frac{\phi_{1}}{\omega_{0}}\left(\bar{a}^{2}+\omega_{1}\right) \sin 2 \bar{\phi}-\frac{\bar{a}^{2} \phi_{1}}{2 \omega_{0}} \sin 4 \bar{\phi} .
\end{aligned}
$$

With the aid of (4.10) and (4.11), and noting that $A_{1}=B_{1}=0$, Eqs. (4.12) and (4.13) can be rewritten as

$$
\omega_{0} \frac{\partial a_{2}}{\partial \bar{\phi}}+A_{2}=\frac{\bar{a}^{5}}{512 \omega_{0}^{3}}(-\sin 2 \bar{\phi}+2 \sin 4 \bar{\phi}+9 \sin 6 \bar{\phi}+\sin 8 \bar{\phi})+\frac{\bar{a} \omega_{2}}{2 \omega_{0}} \sin 2 \bar{\phi}
$$

$\omega_{0} \frac{\partial \phi_{2}}{\partial \bar{\phi}}+B_{2}=\frac{\bar{a}^{4}}{256 \omega_{0}^{3}}(3+5 \cos 2 \bar{\phi}+4 \cos 4 \bar{\phi}+3 \cos 6 \bar{\phi}+\cos 8 \bar{\phi})+\frac{\omega_{2}}{2 \omega_{0}}(1+\cos 2 \bar{\phi})$.

By operating on Eqs. (4.14) and (4.15) with

$$
\frac{1}{2 \pi} \int_{0}^{2 \pi} d \bar{\phi}
$$

and using the assumption that $a_{2}$ and $\phi_{2}$ are periodic in $\bar{\phi}$ with period $2 \pi$, we find that

$$
A_{2}=0, \quad B_{2}=\frac{1}{2 \omega_{0}}\left(\omega_{2}+\frac{3 \bar{a}^{4}}{128 \omega_{0}^{2}}\right) \text {. }
$$


Since $A_{2}=0$, it follows from Eq. (4.5) that correct to $O\left(\varepsilon^{2}\right), \bar{a}$ is a constant and hence to this order $B_{2}$ is a constant. To suppress secular behaviour in the frequency we therefore insist that $B_{2}=0$ and choose $\omega_{2}=-3 \bar{a}^{4} / 128 \omega_{0}^{2}$. We note that, correct to $O\left(\varepsilon^{2}\right), \omega_{2}$ is a constant as required. By integrating the remaining terms in (4.14) and (4.15) with respect to $\bar{\phi}$, we obtain

$$
\begin{aligned}
a_{2}(\bar{a}, \bar{\phi}) & =\frac{\bar{a}^{5}}{1024 \omega_{0}^{4}}\left(7 \cos 2 \bar{\phi}-\cos 4 \bar{\phi}-3 \cos 6 \bar{\phi}-\frac{1}{4} \cos 8 \bar{\phi}\right), \\
\phi_{2}(\bar{a}, \bar{\phi}) & =\frac{\bar{a}^{4}}{512 \omega_{0}^{4}}\left(2 \sin 2 \bar{\phi}+2 \sin 4 \bar{\phi}+\sin 6 \bar{\phi}+\frac{1}{4} \sin 8 \bar{\phi}\right) .
\end{aligned}
$$

To complete the solution we return to (3.2) which, since $\phi=\omega_{0} t+\alpha$, can be written as

$$
u=a \cos \phi \text {. }
$$

Expanding $a$ and $\phi$ in (4.19) according to (4.3) and (4.4) and using (4.10) and (4.11) for $a_{1}$ and $\phi_{1}$ and (4.17) and (4.18) for $a_{2}$ and $\phi_{2}$ we find after a lengthy calculation that correct to $O\left(\varepsilon^{2}\right)$,

$$
u=\left(\bar{a}-\frac{5 \bar{a}^{5}}{4096 \omega_{0}^{4}} \varepsilon^{2}\right) \cos \bar{\phi}+\frac{\varepsilon \bar{a}^{3}}{32 \omega_{0}^{2}} \cos 3 \bar{\phi}+\varepsilon^{2} \frac{\bar{a}^{5}}{1024 \omega_{0}^{4}}(3 \cos 3 \bar{\phi}+\cos 5 \bar{\phi}) .
$$

To obtain $\bar{a}$ and $\bar{\phi}$ we integrate Eqs. (4.5) and (4.6) with respect to $t$ noting that $A_{1}=A_{2}=0$ and $B_{1}=B_{2}=0$ (by choice); this gives

$$
\bar{a}=a_{0}+O\left(\varepsilon^{3}\right), \quad \bar{\phi}=\omega_{0} t+\alpha_{0}+O\left(\varepsilon^{3}\right),
$$

where $a_{0}$ and $\alpha_{0}$ are constants. If we define

$$
b_{0}=a_{0}-\frac{5 a_{0}^{5}}{4096 \omega_{0}^{4}} \varepsilon^{2}
$$

then (4.20) can be rewritten, correct to $O\left(\varepsilon^{2}\right)$, as

$$
\begin{aligned}
u(t ; \varepsilon)= & b_{0} \cos \left(\omega_{0} t+\alpha_{0}\right)+\frac{\varepsilon b_{0}^{3}}{32 \omega_{0}^{2}} \cos \left(3 \omega_{0} t+3 \alpha_{0}\right) \\
& +\frac{\varepsilon^{2} b_{0}^{5}}{1024 \omega_{0}^{4}}\left[3 \cos \left(3 \omega_{0} t+3 \alpha_{0}\right)+\cos \left(5 \omega_{0} t+5 \alpha_{0}\right)\right],
\end{aligned}
$$

and since $\omega_{1}=-3 \bar{a}^{2} / 4$ and $\omega_{2}=-3 \bar{a}^{4} / 128 \omega_{0}^{2}$ it follows by solving (2.5) for $\omega_{0}$ that, correct to $O\left(\varepsilon^{2}\right)$,

$$
\omega_{0}=\left(\omega^{2}+\frac{3 b_{0}^{2}}{4} \varepsilon+\frac{3 b_{0}^{4}}{128 \omega^{2}} \varepsilon^{2}\right)^{1 / 2} .
$$

The solution, given by (4.23) and (4.24), is in complete agreement with the previous solution (2.16) and (2.18). Each solution contains two arbitrary constants which are treated as functions of $\varepsilon$ when imposing the initial conditions. The foregoing analysis was more involved than in Sec. 2 but of course the generalization method of averaging is applicable to a larger class of problems. 
In general, it follows from Eq. (4.6) that if the coefficients $B_{n}(\bar{a})$ for all $n \geqslant 1$ are non-zero constants then $\bar{\phi}$ will be a power series in $\varepsilon$ multiplying $t$ and hence the frequency will exhibit secular behaviour. The procedure to follow would then be to choose, if possible, the $\omega_{n}$ to eliminate the $B_{n}$. Since $B_{n}$ can depend on $t$ only through $\bar{a}$, there will be two classes of problems for which the $B_{n}$ will be constant. Firstly, if $A_{n}=0$ for all $n \geqslant 1$, then it follows from Eq. (4.5) that $\bar{a}$ will be constant. We have seen that the Duffing equation belongs to this class, at least to $O\left(\varepsilon^{2}\right)$. Secondly, if the non-periodic terms in the perturbation equations for $\partial \phi_{n} / \partial \bar{\phi}$ do not depend on $\bar{a}$, then $B_{n}$ will be independent of $\bar{a}$ and hence constant. An example of a problem in this class is the linear oscillator with viscous damping:

$$
\ddot{u}+\omega^{2} u=-2 \varepsilon \dot{u} .
$$

With Eq. (4.25) it is easily verified that $A_{1}=-\bar{a}, A_{2}=0$, so that $\bar{a}$ is not constant, but the perturbation equations for $\partial \phi_{1} / \partial \bar{\phi}$ and $\partial \phi_{2} / \partial \bar{\phi}$ are independent of $\bar{a}$ and the constants $B_{1}$ and $B_{2}$ can be eliminated by choosing $\omega_{1}=0, \omega_{2}=1$. The solution obtained to $O\left(\varepsilon^{2}\right)$ using this modification of the generalized method of averaging is precisely the exact solution, which was also obtained by Veronis [1] using a similar modification to the method of multiple scales. If the coefficients $B_{n}(\bar{a})$ depend on $t$ then in general it will not be possible to eliminate them by suitable choices of the constants $\omega_{n}$. However, if the $B_{n}$ are not constant, then integration of Eq. (4.6) will not result in $\bar{\phi}$ assuming the form of a power series in $\varepsilon$ multiplying $t$, and hence it may not be necessary or desirable to set $B_{n}=0$.

5. Concluding remarks. Both the Linstedt-Poincaré method of strained parameters and the method of averaging can be modified to yield more accurate perturbation solutions to certain problems. For a restricted class of problems (possibly a similar class of problems to that which at present is treated by the Lindstedt-Poincare method) it is sufficient to strain only the parameter in the equation (besides the dependent variable $u$ ) to obtain a uniformly valid perturbation solution.

Sometimes nondimensional variables are used which results in parameters not appearing explicitly in the equation(s). Clearly one would proceed by straining the corresponding coefficients in the nondimensional equation(s) and the technique could possibly be extended to straining suitable coefficients which are not directly associated with parameters.

\section{BIBLIOGRAPHY}

[1] G. Veronis, A note on the method of multiple scales, Quart. Appl. Maths. 38, 363-368 (1980)

[2] A. H. Nayfeh, Perturbation methods, Wiley, New York, 1973, pp. 165, 168

[3] N. Krylov and N. N. Bogoliubov, Introduction to nonlinear mechanics, Princton Univ. Press, Princeton, 1947

[4] N. N. Bogoliubov and Y. A. Mitropolsky, Asymptotic methods in the theory of nonlinear oscillations, Gordon and Breach, New York, 1961, p. 412

[5] A. H. Nayfeh and D. T. Mook, Nonlinear oscillations, Wiley, New York, 1979, pp. 51,52

[6] A. H. Nayfeh, Introduction to perturbation techniques, Wiley, New York, 1981, pp. 113, 139 